\title{
Boris Pasternak: Russian Writer in Italy
}

\author{
Yulia Galatenko (Ph.D.) \\ Philologist, associate professor, teacher of Italian language, translator. \\ National Research University Higher School of Economics, Moscow, Russia.
}

\section{Doi:10.5901/ajis.2013.v2n9p304}

\begin{abstract}
Boris Pasternak is a prominent Russian poet and writer, a Nobel laureate in literature (1958), whose life was essentially connected with Italy. In 1957 his novel "Doctor Jivago" was published in Italy in the Feltrinelli publishing house. The appearance of this novel could be compared with an earthquake, a cultural explosion because Italian readers did not expect that "in the table" of soviet writers was hidden such an interesting fenomenon. The novel instantly became a bestseller.

It was an honor for me to participate in the translation and publication of papers written by Italian writers, critics and historians of literature who reacted immediately upon the publication of "Doctor Jivago": e.g., A. Moravia, I. Calvino, F.Fortini, C.Cassola, C.Salinari, etc. In this paper a classification of their opinions, reactions and points of view is presented. These opinions were vivid, natural, not dictated by ideology (as it happened in the URSS). The Italian publishers and Italian critics, who mostly praised the novel, played the sunstantial role in the worldwide advance of the novel "Doctor Jivago". It is interesting that Italian critics (that were not well informed of the soviet literature because it was mainly "hidden in the tables" of soviet writers) evaluated the novel correctly and even guessed the perception of the novel by Russian critics. I'm happy to make a presentation of the book entitled "«Doctor Jivago» Pasternak, 1958, Italy" that was published in Russia. The book was published in Russian, but its edition in the initial language of the italian critics and writers - in Italian - is also indubitably worth publishing.
\end{abstract}

\section{Introduzione}

Nel 1957 in Italia presso la casa editrice "Feltrinelli" è uscito il romanzo di Boris Pasternak "Il Dottor Zivago". Boris Pasternak - famosissimo poeta e scrittore russo del primo Novecento - Ricevette il Premio Nobel per la letteratura quasi subito dopo l'uscita del romanzo " II Dottor Zivago", che fu pubblicato appunto in Italia.

\section{La storia della pubblicazione}

Nella sua patria, in Unione Sovietica, Pastenak come molti altri scrittori subì persecuzioni da parte del governo; tutti erano accusati di diffondere delle idee contro la politica comunista anche se effettivamente non gli passava nemmeno dalla mente di contrastare la politica o combatterla. Si diceva che la letteratura ufficiale sovietica non poteva produrre nulla di buono, perchè l'ispirazione degli scrittori russi era bloccata fin dal principio: gli scrittori non erano liberi di esprimere i propri pensieri, anche se credevano di esserlo. P.e. uno scrittore senza dubbio tipico sovietico - Alexandr Fadeev (conosciuto anche all'estero, anche in Italia) non era più libero di un anticomunista, perchè la sopraffazione ideologica soffocava anche in lui la possibilità di far qualcosa di vivo e di vero.

Nell'Unione Sovietica qualsiasi letteratura veniva punita e soffocata. Perciò molte opere e anche capolavori degli scrittori russi erano "capolavori nel cassetto" come si diceva, cioè nascosti, non pubblicati. La stessa cosa succedeva anche a Pasternak, che però riuscì a pubblicare il suo romanzo all'estero, in Italia in lingua italiana. I burocrati sovietici non solo non hanno permesso la pubblicazione del libro in patria, ma hanno tentato persino di impedirne la pubblicazione all'estero. Si legge in C. Cassola: "così facendo, hanno reso un pessimo servizio non soltanto al proprio paese (un libro come il "Dottor Zivago" giova più al prestigio culturale della Russia di cinquanta Spuntnik), ma allo stesso regime sovietico", perchè il romanzo di Pasternak non è per niente un romanzo sovietico [7; 534]. La stessa cosa facevano anche i comunisti e sovietici italiani, facevano il possibile per impedire l'uscita del romanzo di Pasternak anche in Occidente. II romanzo venne rifiutato dall'Unione degli Scrittori dell'Unione Sovietica, che ai tempi del regime comunista non poteva permettere la pubblicazione di un libro, senza alcun dubbio autobiografico, che raccontava i lati più oscuri della Rivoluzione d'ottobre. II romanzo diventò la causa di persecuzioni dello scrittore da parte del regime e dei servizi segreti che lo costrinsero alla povertà e al totale isolamento. Infatti Boris Pasternak morì pochi anni dopo l'uscita del romanzo nella sua casa a Peredelkino completamente solo. 
Però il romanzo si diffuse in occidente e nel giro di pochissimo tempo venne tradotto in molte lingue, diventando la vera testimonianza della realtà sovietica.

Nel 1964 il poeta Eugenio Montale scrisse le parole iniziali della prefazione a "Il dottor Živago": "Non occorreva conoscere la lingua russa per sapere che Pasternak occupava un posto considerevole tra i moderni lirici russi: un posto tanto importante da far sì che molte delle sue poesie fossero tradotte nelle varie lingue europee assai prima che venisse alla luce "Il dottor Živago" [12].

In Russia legalmente il romanzo fu pubblicato soltanto nel 1988 (28 anni dopo la morte di Pasternak), pochi anni prima della caduta dell'Unione Sovietica, quando le riforme di Gorbačëv fecero respirare un po' di libertà.

\section{Pasternak e il Premio Nobel}

Nel 1958 il "Dottor Zivago" fruttò a Pasternak l'assegnazione del Premio Nobel per la letteratura. Ma la storia di tutto questo è molto tragica e spiacevole. Pasternak inviò un telegramma a Stoccolma all'Accademia Svedese per esprimere la propria gratitudine, però alcuni giorni più tardi fu costretto a scrivere la sua rinuncia. Infatti Pasternak subì pressanti minacce e avvertimenti dalla parte del KGB: dalla sua definitiva espulsione dalla Russia fino alla confisca delle proprietà (già troppo limitate). In questo modo lo costrinsero a rifiutare la fama e il riconoscimento che avrebbe trovato all'estero. Solo dopo la rinuncia la persecuzione finì. Così non ritirò mai il premio. Morì due anni più tardi in povertà nella sua casa a Peredelkino, nei dintorni di Mosca, nel 1960.

E solo nel 1989 il figlio dell'autore Evgenij Pasternak si recò in Svezia per ritirare il Premio spettante a suo padre 31 anni prima.

\section{Opere conosciute in Italia prima del "Dottor Zivago"}

Le prime opere del poeta russo arrivarono in Italia molto prima del 1957, già negli anni venti, periodo dell'avvio degli studi di slavistica in Italia per merito di preziosi studiosi: Ettore Lo Gatto, Renato Poggioli, Giovanni Maver e con la collaborazione dell'emigrazione russa [cfr. 5]. Le opere di B. Pasternak vennero ampiamente tradotte in italiano, perciò possiamo considerare Pasternak un fenomeno anche della letteratura italiana. Raissa Naldi nel 1905 fu la curatrice della prima antologia di poeti russi contemporanei - del 1924 - nella quale sono contenute alcune poesie di Pasternak. La "più grande promessa della nuova poesia russa" - così venne definito Pasternak nell'antologia del 1933 dal titolo "La violetta notturna" [11], nella quali sono presentati i più importanti poeti russi, tra cui: Aleksander Blok, Nikolaj Gumelev, Osip Mandelštam, Vladimir Majakovskij, Anna Achmatova, Marina Cvetaeva. Poi una successiva antologia del 1949 dal titolo "Il fiore del verso russo" [6] fu curata da Renato Poggioli, dove emerge l'immagine di un poeta mosso da istanze romantiche, pulsioni, passioni e resistenze, legato alla sensibilità di poeti classici: Lermontov e di Fet. C'è anche da segnalare una raccolta di poesie di Pasternak, pubblicata nel volume "Autobigrafia e nuovi versi" e il fatto molto importante, è che riuscì a pubblicarla per la prima volta solo in Italia.

Durante la seconda guerra mondiale, con le pressioni della censura fascista, come nota E. Baglioni, "Io studio della letteratura russa subisce un generale rallentamento, in particolare l'ambito contemporaneista ${ }^{1}$, percepito come propaggine dell'ideologia socialista. Esso riprenderà con vivacità solo nel tardo dopoguerra". E solo nel 1945 Pasternak apparve di nuovo, in questo caso attraverso il traduttore Pietro Zveteremich, e venne presentato come poeta interno al clima rivoluzionario e sovietico.

Nel 1946 nella rivista di Elio Vittorini "II Politecnico" apparve per la prima parte del "Salvacondotto" - un libro autobiografico, dedicato ai ricordi.

Nel 1957 oltre alle ben note vicende legate al romanzo, in Italia esce la pubblicazione presso Einaudi del primo volume interamente dedicato alle poesie di Boris Pasternak [15]. L'operazione editoriale compiuta da Einaudi è il frutto di un lavoro di esegesi e traduzione intrapreso da un noto professore dell'Università La Sapienza di Roma: Angelo Maria Ripellino. Ripellino inizia ad occuparsi di Pasternak dalla fine degli anni Quaranta, continua a tradurlo includendo sue

\footnotetext{
${ }^{1}$ De Michelis ricorda tre episodi sintomatici del clima culturale repressivo: la pubblicazione nel 1939 degli Elenchi di opere la cui pubblicazione, diffusione e ristampa nel Regno è stata vietata dal Ministero della cultura popolare, che avevano proibito qualsiasi materiale provenisse dall'Unione Sovietica; l'emigrazione nel 1938 del più importante russista in Italia, Renato Poggioli, e l'incarcerazione e la morte dello specialista di letteratura russa e traduttore Leone Ginzburg. [Cfr. 4, 209].
} 
opere nella "Poesia russa del Novecento" [16] del 1954 (Feltrinelli, Milano, 1960, 1973), fino a raccoglierne una cospicua selezione monografica nel 1957.

Tutto sommato Boris Pasternak era già noto e conosciuto in Italia anche prima dello scandalo intorno al romanzo "Il Dottor Zivago", benchè (come diceva il traduttore del romanzo in lingua italiana - Pietro Zveteremich [19]), fosse noto piuttosto al "pubblico d'élite".

\section{Slavisti italiani e Boris Pasternak}

Pasternak era noto non solo ai lettori italiani, ma piuttosto ai critici. Anche prima dell'uscita del "Dottor Zivago" Pasternak era molto stimato dagli slavisti italiani. Tra i più celebri c'era Vittorio Strada, che da molti anni era docente all'Università Ca' Foscari di Venezia, ex direttore dell'Istituto Italiano di Cultura di Mosca. All'età di 83 anni Vittorio Strada è considerato ancora oggi uno dei più grandi critici e slavisti italiani, fra i massimi esperti del mondo russo e della sua indecifrabile "anima russa". Ha dedicato una grande parte della sua ricerca proprio alla persona di Boris Pasternak, ha curato una notevole raccolta di saggi "I russi e l'Italia" [8]. V. Strada si è seduto a tavola con i più grandi scrittori del XX secolo e ha brindato con Pasternak nella sua casa a Peredelkino. Era Vittorio Strada ad aiutare la pubblicazione del romanzo di Pasternak in Italia.

Un altro noto critico e slavista, Sergio d'Angelo, giocò un ruolo importantissimo per Boris Pasternak e il suo romanzo. D'Angelo era un giornalista italiano con un incarico di redattore inviato da Radio Mosca in sezione per le trasmissioni in Italia proprio nel periodo dello scandalo legato al romanzo di Pasternak. Nel maggio del 1956 d'Angelo incontrò Boris Pasternak, da cui ricevette l'inedito testo del romanzo con l'intesa di affidarlo all'editore italiano Feltrinelli. Sergio d'Angelo servì da collegamento tra Pasternak e la casa editrice italiana, un filo in questo lungo percorso del romanzo.

Uno degli specialisti più assidui fu Cesare G. De Michelis, l'autore di un libro dedicato a Pasternak. Tendeva a sottolineare che benché Pasternak avesse trascorso in Italia solo un breve periodo nell'adolescenza, l'Italia fu destinata ad avere un gran peso nella vita del poeta perchè proprio in Italia nacque la sua fama come scrittore $[3,6]$. Vero è che il nome di Boris Pasternak diventò famoso grazie allo scandalo e alla fama sensazionale dovuta alla storia de creazione del romanzo.

\section{Reazione dei critici}

Subito dopo la pubblicazione del romanzo di Pasternak in Europa avvenne un'esplosione culturale; Pasternak ha messo in moto il campo letterario italiano: molti famosi scrittori e critici lettarari espressero il desiderio di esprimere la propria opinione nei confronti del romanzo. Bisogna renderci conto del fatto che quasi tutti pensavano, che la letteratura russa fosse destinata a morire di morte naturale per i motivi politici, e l'apparizione del romanzo il "Dottor Zivago" fu veramente una sorpresa. Che Pasternak sia sopravissuto e sia riuscito a scrivere il "Dottor Zivago" dimostra che lo stalinismo ha causato l'arresto ma non l'interruzione definitiva o la scomparsa della tradizione narrativa russa.

Grazie al lavoro molto duro e scrupoloso di Alessandra Reccia, che aveva ritrovato tanti articoli di giornali e di riviste italiani scritti dagli scrittori e scienziati più notevoli per far vedere la loro reazione all'uscita del romanzo, noi abbiamo potuto pubblicare questi saggi in lingua russa. E' veramente una cosa meravigliosa che un numero così grande di studiosi italiani abbia manifestato il desiderio di fare la propria ricerca, di esprimersi, di dire la sua in proposito.

Questo libro è stato una vera sconcertante e splendida sorpresa. Prima di leggerlo molti lo ritevano un pamphlet antistalinista. Prima del romanzo di Pasternak la critica letteraria italiana conosceva pochi esempi della narrativa russa solo "Nella città natale" di Nekrasov, "II Disgelo" di Ehrenburg, "Non si vive di solo pane" di Dudintzev. Ma "Dottor Zivago" non ha niente a che fare con queste opera e con quello che veniva pubblicato in Russia in quel periodo. In Europa si parlava sopratutto della crisi del romanzo come tale, sostenendo la fine del romanzo o la sua trasformazione in un documento o saggio di genere filosofico. Inoltre molti critici italiani erano abituati da decenni a pensare alla grande narrativa russa come ad un fenomeno limitato all'Ottocento, che si era chiusa con Cecov. Anzi si riteneva che la letteratura russa fosse morta non solo per la pressione esercitata dallo stalinismo, ma piuttosto di una morte naturale, benchè ci si rendesse conto della forte pressione ideologica-politica del regime che realmente aveva falsato il corso della letteratura russa, avendo soffocato in germe dei possibili capolavori.

Ci sono diversi tipi di giudizi. Due primi approcci rispecchiavano due atteggiamenti completamente diversi nei confronti del romanzo. Una parte dei critici lo lodava e lo portava alle stelle, l'altra invece lo biasimava e non ne vedeva niente di interessante e nuovo. 
Il primo esercito ha proclamato il romanzo "il più bel romanzo del secolo" [7,. 529], Guglielmo Petroni: "considero il "Dottor Zivago" un'opera molto bella", "è un libro innovatore", "progressista" [7, 711-712]. Venivano pronunciati i giudizi estremamente forti. Per esempio, come quello di Carlo Cassola, che "di libri-chiave tipo "L'étranger" o "La nausée" o "Il dottor Faustus" se ne potrebbe fare benissimo a meno", "è chiaro che con tutta questa roba il "Dottor Zivago" non ha assolutamente niente in comune" [7, 532]. Cassola tendeva a sottolineare "la freschezza, la luminosità, la genuità, la nativa, naturale poeticità di questo romanzo" [7,.529]. "Pasternak fa benissimo a seguire solo la legge della sua ispirazione" [7, 531].

Italo Calvino ammirava il respiro e l'altezza del romanzo [7, 717]. G.B. Angioletti definiva il "Dottor Zivago" "un'opera magnifica, dove si ritrovano intatti gli elementi poetici, epici e morali che consentirono la grandezza passata della letteratura russa" [13]. G. Piovene nel romanzo ravvisava "una novità, una scoperta e un punto d'arrivo" di tutta la letteratura occidentale [14].

Tra i primi era Pietro Citati che vedeva nel "Dottor Zivago" un vero e proprio "romanzo" intimamente sorretto e articolato da una schematica costruzione" [18].

II secondo esercito si inclinava verso il giudizio fortemente limitativo o addirittura negativo. Molti critici ammettevano che le descrizioni di paesaggio e tutta la storia d'amore fossero molto belle, però nel suo insieme sarebbe stato da considerarsi mancato perché il romanzo è scritto da un lirico a cui manca il fiato per una vasta rappresentazione narrativa. Quelli che rifiutavano a priori il romanzo di Pasternak avevano la stessa opinione, che il romanzo fosse un genere letterario con delle strutture fisse, ossia fissate dai grandi scrittori dell'Ottocento, e i personaggi dovessero essere ben elaborate e precisi (la pensava così, p.e., Herling). Ma sicuramente Jurij Zivago non è un personaggio del tipo di quelli di Leo Tolstoj - il principe Andrea ecc. E' chiaro che se si confronta con personaggi classici di Tolstoj o Dostievskij, Lara appare sfuocata e Juriy spezzato. Se si rimane fermi agli schemi romanzeschi dell'Ottocento, usando i termini, le definizioni adeguate solo ai romanzi classici, allora sì che il "Dottor Zivago" non ha personaggi, non ha unità, non ha coesione di svolgimento ecc.

Cesare Cases non riteneva il "Dottor Zivago" un romanzo veramente grande, perchè non dà "la sintesi di un'epoca" [7,. 845].

Alberto Moravia diceva che "sono stati pubblicati libri di narrativa più belli o più importanti del "Dottor Zivago"'” [7, 843], tra i quali "La Rechèrche" di Proust, "L'Ulysses" di Joyce, i libri di Svevo, Mann, Kafka, Hemingway, Woolf ecc.

II critico C. Salinari dichiara di preferire il "Placido Don" di Sciolochov [1]. Secondo Salinari il libro di Pasternak fallisce come romanzo ogni volta che si incontra con la storia perché si muove nell'ambito di una poetica individualistica.

Riccardo Bacchelli ha dichiarato esplicitamente le proprie limitazioni e i propri dubbi circa la validità del "Dottor Zivago", avendo scoperto nel libro un vizio stilistico e un difetto tecnico. II primo consisteva, secondo il critico, nella sovrabbondanza di figure e immagini ricercate e preziose, quasi barocche; il secondo era nell'insistenza su incontri e ritrovamenti troppo straordinari e meravigliosi.

Io ho avuto la fortuna di fare la traduzione di una gran parte di articoli e saggi di molti critici letterari italiani della metà del Novecento [20], tra cui il saggio più importante e significativo è sicuramente il questionario proposto ai critici dalla rivista "II Ponte". Dall'aprile del 1958 "II Ponte" ha dato l'avvio all'inchiesta dichiarando la curiosità che si è condensata intorno al libro. Nel determinare i temi della discussione la redazione del "Ponte" ha scelto delle domande interessanti come test per le reazione di ambiente. L'inchiesta era aperta sui seguenti punti: 1) E' il "Dottor Zivago" il romanzo del secolo? 2) II "Dottor Zivago" e il decadentismo; 3) e la tradizione russa; 4) e la letteratura sovietica. A tali quesiti hanno risposto numerosi scrittori e critici, tra i quali: G.B. Angioletti, A. Banti, I. Calvino,M. Cancogni, C. Cases, C. Cassola, P. Citati, F. Fortini, T. Landolfi, A. Moravia, G. Piovene, C. Salinari ecc.

Alcuni critici (G. Petroni e molti altri) consideravano il "Dottor Zivago" un romanzo che avesse "legami con la tradizione aulica del proprio paese ma che al medesimo tempo rompa quella tradizione" [7, 713].

A proposito del romanzo il "Dottor Zivago" ci si ricollega sempre ai romanzi di L. Tolstoj, e spesso non a favore dell'opera pasternakiana. I critici vedevano le stesse concezioni da entrambi gli scrittori, però nei confronti di "Guerra e pace" il "Dottor Zivago" sembra "decisamente un romanzo mancato" [7, 845]. Molti (tra cui Manlio Cancogni) ritenavano ovvio che Pasternak non scrivesse come Tolstoj e che il "Dottor Zivago" "non ha il ritmo e il respiro di "Guerra e pace". Pasternak veniva percepito esclusivamente come un lirico, per il quale la realtà "è un susseguirsi di momenti lirici" [7, 714]. Cesare Cases addirittura tendeva a sottolineare che "là dove fallisce il Pasternak romanziere soccorre il Pasternak lirico, con la sua forte vitalità e concretezza" [7, 846].

E' chiaro che tutti mettevano il romanzo nel contesto della tradizione narrativa classica. Italo Calvino ha definito meglio di tutti che "Il "Dottor Zivago" è un romanzo del Novecento che sviluppa la tradizione russa ottocentesca nella forma (Tolstoj, Cecov, il loro modo di voler raccontare la vita; non Dostojevskij) e nella sostanza (Tolstoj, Dostojevskij, non 
Pare che conoscessero soltanto tre scrittori classici russi con i quali facevano sempre il paragone, usandoli come l'unico punto di riferimento.

Qualcuno ha definito il "Dottor Zivago" un romanzo decadente dopo avervi incontrato alcuni tipici aspetti e difetti del decadentismo: frammentismo, allusività, dissolvimento dell'lo ecc. P.e. Cesare Cases chiamava il romanzo di Pasternak "tipicamente ispirato ai moduli dell'arte decadente" $[7,846]$ per quanto riguarda la composizione. A parere di Alberto Moravia il "Dottor Zivago" "è un romanzo composito nel quale si fondono la tradizione classica e oggettiva (Puschkin, Tolstoj, Cecov) della narrativa russa e il decadentismo europeo" [7,. 843]. Però Italo Calvino, p.e., ha rinunciato a usare la parola "decadentismo" non solo nei confronti di Pasternak, ma anche in generale, per un motivo abbastanza banale che non "ha ancora capito bene che cosa sia, e se sia cosa da biasimarsi o da lodarsi" [7, 717].

Per quanto riguarda la quarta domanda, ossia la posizione del romanzo di Pastermak in conformità con la tradizione sovietica, è chiaro che molti critici e saggisti italiani hanno preferito non rispondere in quanto non si ritenevano competenti in questione. Infatti negli anni '50 la letteratura sovietica non era assai nota all'estero (salvo l'opera di Sciolokhov, Didinzev e alcuni altri scrittori), perciò il contesto sul quale è apparso il romanzo di Pasternak in Italia non era ben chiaro e preciso. Alcuni critici letterari però (Cesare Cases) ne cercavano i tratti antisovietici e ne hanno trovati pochi. "Se non è un romanzo antisovietico, è certo un romanzo che rifiuta, sia nelle concezioni del mondo, sia nell'espresione letteraria, tutte le tendenze del pensiero, ufficiali o meno, legate all'esistenza della società e dello Stato sovietico" [7, 849]. Cases in effetti aveva "un sentimento di viva irritazione", che "debba succedere a chiunque condivida le concezioni e le prospettive del socialismo" [7, 849].

Quanto a Cecov, il critico e lo scrittore famoso Carlo Cassola racconta che gli è venuta in mente la vignetta comparsa all'epoca di disgelo in un giornale russo, nella quale gli scrittori sovietici facevano anticamera davanti al gabinetto del dott. Cecov; e lui emetteva diagnosi spietate e prescriveva rimedi radicali. Questa vignetta metteva a nudo quello che succedeva con la letteratura sovietica, i cui prodotti erano banali, piatti, schematici, assolutamente indegni della grande tradizione narrativa russa. L'unico rimedio che si sapesse proporre era rivolgersi al dott.Cecov, ma Cecov non poteva più dare ricette per la salute della letteratura contemporanea.

Sta di fatto che non si trattava di rifare Tolstoj o Cecov, ma di fare opere nuove, vive, poetiche - e questo voleva dire continuare la tradizione vera della narrativa classica russa: ciò che appunto ha fatto Pasternak nel suo romanzo il "Dottor Zivago", continuando il filone Tolstoj-Cecov.

Mi sembra un giuduzio molto importante e molto profondo quello di C. Cassola sulla letteratura russa in generale. Lui continuava a ripetere che in Russia la letteratura aveva sempre un'importanza incomparabilmente maggiore che nell'Occidente. In Russia gli scrittori erano sempre i soli direttori di coscienza della, mentre in Europa "questo ruolo è stato assunto piuttosto dai filosofi" [7, 534]. La opinione di Cassola è molto interessante, ritiene che "il colpo mortale all'autocrazia zarista gliel'ha inferto la letteratura" $[7,534]$ - proprio così si spiega come la nuova autocrazia sovietica abbia tutte le ragioni di temere la letteratura e quindi di soffocarla.

C'è la sensazione che Pasternak sia appartenuto a due culture - quella russa e quella italiana. E non solo grazie allo scandalo intorno al "Dottor Zivago". Lui faceva parte del circuito futuristico ed ermetico e si sentiva vicino ai crepuscolari italiani. E' possibile fare molti altri paragone tra l'opera di Pasternk e quella degli scrittori italiani. Per esempio, lo stesso protagonista Juriy Zivago - una persona creativa che diventa giudice del proprio tempo - e il romanzo di Antonio Fogazzaro "Piccolo mondo antico", "Il piacere" di Gabriele D’Annunzio, il dramma i "Giganti della montagna" di Luigi Pirandello. "Il Dottor Zivago" assomiglia anche ai romanzi italiani, scritti più o meno nello stesso tempo del romanzo di Pasternak - quelli di Elio Vittorini, Cesare Pavese, Italo Calvino - in quanto danno un quadro del paese in via di cambiamento. In altre parole, Boris Pasternak entrò nel contesto della cultura italiana con molta disinvoltura.

Alla fine bisogna dire che il "Dottor Zivago" non si presta a ricevere un'etichetta, a essere catalogato, classificato o assegnato a questa o quella tendenza letterario. II romanzo di Pasternak è un vero miracolo con il suo specifico fascino slavo, ma con un destino italiano.

\section{References}

"Contemporaneo" 19 genn. 1958

D’Angelo S. II caso Pasternak. Milano, 2006.

De Michelis C.G. Boris Pasternak. Poesie. Torino, 1992.

De Michelis C.G., "Letteratura russa del Novecento", in La slavistica in Italia. Cinquant'anni di studi (1940-1990), p.209.

Diddi C., "La slavistica italiana del primo dopoguerra nella rivista "i libri del giorno (1918-1929)", in Europa Orientalis 27, 2008.

Il fiore del verso russo, a cura di Renato Poggioli, Einaudi, Torino 1949.

"Il ponte", aprile 1958.

I russi e L'Italia. A cura di Vittorio Strada. Libri Scheiwiller, Milano, 1995. 
Lo Gatto E. I russu in Italia. Roma, 1971.

Lo Gatto E. Storia della letteratura russa contemporanea. Milano, 1958.

La violetta notturna, a cura di Renato Poggioli, Carabba, Lanciano 1933

Montale E., Prefazione, in B. Pasternak, Il dottor Živago, Einaudi, Torino 1964.

"Nuova Stampa" 8 febbr. 1958.

"Nuova Stampa" 19 febbr. 1958.

Pasternak B., Poesie, a cura di A.M. Ripellino, Einaudi, Torino 1957.

Ripellino A.M,, Poesia russa del Novecento, Guanda, Parma 1954.

Strada V. La questione russa. Identità e destino. Venezia, 1991.

"Tempo presente", aprile 1958

Zveteremich P. Gli anatemi per Zivago. II centenario della nasita di Pasternak // La Sicilia. 15.02.1990.

«Доктор Живаго»: Пастернак, 1958, Италия. М.: Река времен, 2012. 